DOI: $10.2478 /$ ace-2014-0027

\title{
NUMERICAL PREDICTION OF THE EFFECT OF LAMINATION ORIENTATION ON FRACTURE BEHAVIOUR OF WIRES FOR CIVIL ENGINEERING APPLICATIONS
}

\author{
K.K. ADEWOLE ${ }^{1}$ AND S.J. BULL ${ }^{2}$
}

\begin{abstract}
This paper presents a numerical investigation of the effects of lamination orientation on the fracture behaviour of rectangular steel wires for civil engineering applications using finite element (FE) analysis. The presence of mid-thickness across-the-width lamination changes the cup and cone fracture shape exhibited by the lamination-free wire to a V-shaped fracture with an opening at the bottom/pointed end of the V-shape at the mid-thickness across-the-width lamination location. The presence of mid-width across-the-thickness lamination changes the cup and cone fracture shape of the lamination-free wire without an opening to a cup and cone fracture shape with an opening at the lamination location. The FE fracture behaviour prediction approach adopted in this work provides an understanding of the effects of lamination orientation on the fracture behaviour of wires for civil engineering applications which cannot be understood through experimental investigations because it is impossible to machine laminations in different orientations into wire specimens.
\end{abstract}

Keywords: Finite element, Fracture behaviour, Lamination orientation, Wire

\section{INTRODUCTION}

Failure analyses of wires used for civil engineering applications, such as the failure analysis of the fractured pre-stressing wires of the ruptures Jordan Aqueduct, Reach 3 Pre-stressed Concrete Pipes conducted by the United States Bureau of Reclamation [1] and the failure analysis of the broken wires of a suspension bridge conducted by Mahmoud [2] are designed to identify the cause(s) of the failure of the wires in order that appropriate corrective action may be taken. The study of the fracture behaviour/ performance and failure analysis of wires used for civil engineering applications, such as the studies of the fracture behaviour of pre-stressing wires conducted by Toribio and Ayaso [3, 4] and Toribio and Valiente [5] are also conducted on experimental specimens of pre-service wires for quality control purposes Vander Voort [6].

1 School of Chemical Engineering and Advanced Materials, Newcastle University, Newcastle upon Tyne, United Kingdom, NE1 7RU, e-mail: kkadewole@yahoo.com

2 School of Chemical Engineering and Advanced Materials, Newcastle University, Newcastle upon Tyne, United Kingdom, NE1 7RU, e-mail: s.j.bull@ncl.ac.uk 
Wires for civil engineering applications, as all engineering materials, are not defect-free and often contains defects such as notches/scratches, cracks, laminations etc, which may affect their fracture performance and threaten their structural integrity. Consequently, for the structural integrity of wire reinforced or wire pre-stressed structures, where wires serve as the load carrying member or contribute to the load carrying capability of the structure, an understanding of the effects of defects on the fracture behaviour of wires is essential. There is a need to have an understanding of the effect of laminations in particular on the fracture behaviour of rolled wires. For example, the presence of laminations in the Jordan Aqueduct, Reach 3 Pre-stressed Concrete Pipes pre-stressing wires has been identified as the cause of the failure of the concrete pipes/ aqueduct [1]. Laminations are usually invisible, long linear crack-like defects that are usually parallel to the wires' rolling or drawing direction Peet and Wilde [7]. One of the techniques for the detection of laminations in wires is by subjecting the wire to a reverse bending and straightening test. However, Adewole and Bull [8] have revealed that mid-width laminations in wires subjected to the reverse bending and straightening test may go undetected. The laminations that were responsible for the failure of the Jordan Aqueduct, Reach 3 Pre-stressed Concrete Pipe pre-stressing wires were found to be randomly oriented around the wire's circumference. For rectangular wires, the laminations could be orientated across-the-width and/or across-the-thickness of the wires. Consequently, there is the need for the understanding of the effects of lamination orientation on the fracture behaviour of rectangular wires.

Experimental investigations of the effects of notches, vertical surface cracks and the number of drawing passes on the fracture behavior of wires for civil engineering applications have been conducted by Toribio and Ayaso [3], Toribio and Ayaso [4], and Toribio and Valiente [5] respectively. While notches can be machined into wires, surface cracks can be experimentally introduced into wires by axial fatigue loading, and the number of drawing passes the wires undergo can be experimentally simulated, the longitudinal mid-thickness across-the-width and mid-width across-the-thickness linetype/crack-like laminations such as those that were responsible for the failure of the Jordan Aqueduct, Reach 3 Pre-stressed Concrete Pipe pre-stressing wires cannot be experimentally machined into wire specimens. Thus, alternative approaches are needed to study and understand the effect of the orientation of the line-type/crack-like laminations on the fracture behaviour of rectangular wires. In this paper, the effects of the orientation of longitudinal linear crack-like laminations on the fracture behaviour of a typical rectangular carbon steel wire used for civil engineering applications is investigated using Finite Element (FE) analysis. The investigation covers the effects of longitudinal mid-thickness across-the-width and mid-width across-the-thickness linear crack-like laminations on the fracture behaviour (fracture process sequence and fracture shape) of rectangular steel wires. 


\section{EXPERIMENTAL}

Test samples were made from a low carbon manganese steel wire material $(0.18 \% \mathrm{C}$, $0.6 \% \mathrm{Mn}$ ). Laboratory tensile testing was conducted on two wire sizes with $12 \mathrm{~mm} \mathrm{x}$ $5 \mathrm{~mm}$ and $12 \mathrm{~mm} \times 7 \mathrm{~mm}$ cross-sectional dimensions. For each wire size, ten full-size lamination-free wire specimens with an overall length of $170 \mathrm{~mm}$ and a gauge length of $50 \mathrm{~mm}$ shown in Figure 1 were tested. The tensile test was conducted with an Instron universal testing machine (IX 4505) with a static capacity of $\pm 100 \mathrm{kN}$. The specimens were held with wedge grips and the displacement of the $50 \mathrm{~mm}$ gauge length was measured with an Instron 2630-112 clip-on strain gauge extensometer with a $50 \mathrm{~mm}$ gauge length. The tensile tests were conducted on lamination-free wire specimens at a cross head speed of $5 \mathrm{~mm} / \mathrm{min}$, an ambient temperature of $20^{\circ} \mathrm{C}$ and a relative humidity of $38 \%$. No tensile test was conducted on wire with laminations because as earlier stated, it was not experimentally possible to machine the long longitudinal mid-thickness across-the-width and mid-width across-the-thickness linear crack-like laminations such as those responsible for the failure of the pre-stressing wires of the ruptured Jordan Aqueduct pre-stressed concrete pipes into the wires.

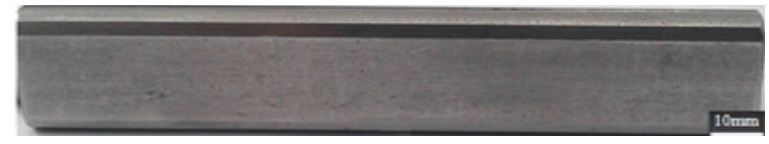

Fig. 1. Experimental wire specimen

\section{Finite ElEMENT TENSILE TESTING SIMULATION}

The FE simulations of the tensile test, elastic, plastic and fracture behaviours of the wires were conducted with the isotropic elastic-plastic model and the micromechanical-based phenomenological shear failure fracture model. This combination has been established to be a suitable model combination for the accurate prediction of the entire elastic, plastic and fracture behaviours of the typical wire for civil engineering applications used for this work by Adewole [9]. Details of the models and their parameters can be obtained from Adewole [9]. Taking an advantage of the symmetry of the wires, the simulations of the tensile testing of the wire with and without laminations were conducted on half models of the wires with and without laminations as shown in Figures 2(a) to (c) to reduce the FE computation time and the FE results' file size. The $10 \mathrm{~mm}$ long longitudinal mid-thickness across-the-width and mid-width across-the-thickness line-type/crack-like laminations are not visible in meshed models in Figure 2(a) as they were modelled as seams (partition lines which open during analysis) that have been established in Adewole [10] to be an appropriate modelling technique for the simu- 
lation of the linear crack-like laminations considered in this work. Consequently, the wireframe images of the wire models with and without laminations are also provided in Figures. 2(b) to (d).

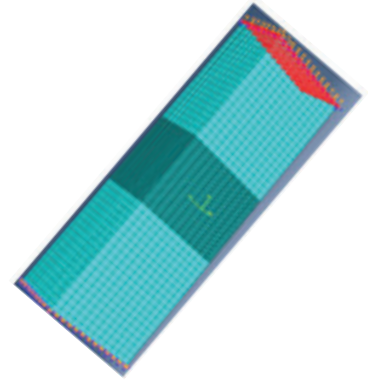

(a) Meshed wire model

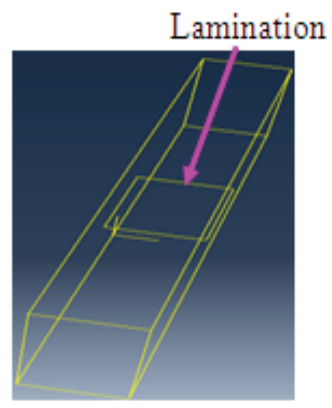

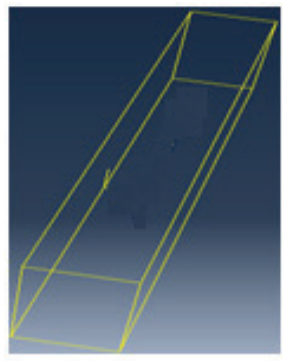

(b) Lamination-free

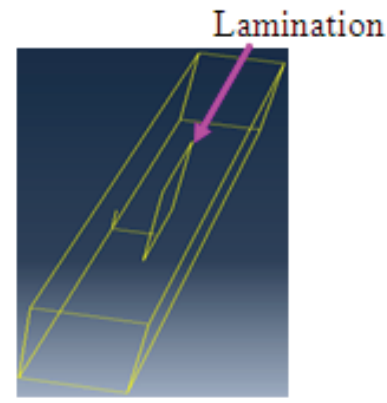

(c) Mid-thickness lamination (d) Mid-width lamination

Fig. 2. FE models of wires with and without laminations

The models of the wire specimens were meshed with C3D8R (8-node linear brick, reduced integration, hourglass control) elements of two sizes: $1 \mathrm{mmx} 1 \mathrm{mmx} 1 \mathrm{~mm}$ and $0.1 \mathrm{~mm} \times 0.1 \mathrm{~mm} \times 1 \mathrm{~mm}$ elements. The outer regions of the models of the wire specimens with the mid-thickness across-the-width lamination, mid-width acrossthe-thickness laminations and the lamination-free wire were meshed with the coarse $1 \mathrm{mmx} 1 \mathrm{mmx} 1 \mathrm{~mm}$ elements, while the middle region of the three wire models was meshed with the fine $0.1 \mathrm{~mm} \times 0.1 \mathrm{~mm} \times 1 \mathrm{~mm}$ elements as shown in Figure 2 (a). The fine elements were positioned with their $1 \mathrm{~mm}$ dimension in the global direction of the wire's width. This element size combination and orientation has been established as the optimum mesh/element size combination and the appropriate element orientation for 
the accurate prediction of the tensile and fracture behaviours of the wires considered in this work by Adewole [11]. The left hand end of the models was completely fixed and the right hand end was partially fixed without any restraint to its translational movement in the wire's longitudinal direction. The partially fixed right hand end of the model was subjected to a longitudinal displacement.

\section{RESULT}

The FE predicted deformed shapes and fracture process sequences for the two wire sizes are similar, consequently, only the deformed shapes and fracture process sequence for the $12 \mathrm{mmx} 7 \mathrm{~mm}$ wire are presented. The experimental and FE force-displacement curves for the lamination-free wire are presented in Figure 3. The FE predicted deformed shapes for the lamination-free wire, the wire with the mid-thickness acrossthe-width lamination and the wire with the mid-width across-the-thickness laminations after necking are shown in Figure 4. The FE predicted deformed shapes throughout the fracture process of the lamination-free wire, the wire with the mid-thickness across-thewidth lamination and the wire with the mid-width across-the- thickness lamination are presented in Figures 5, 6, and 7 respectively.

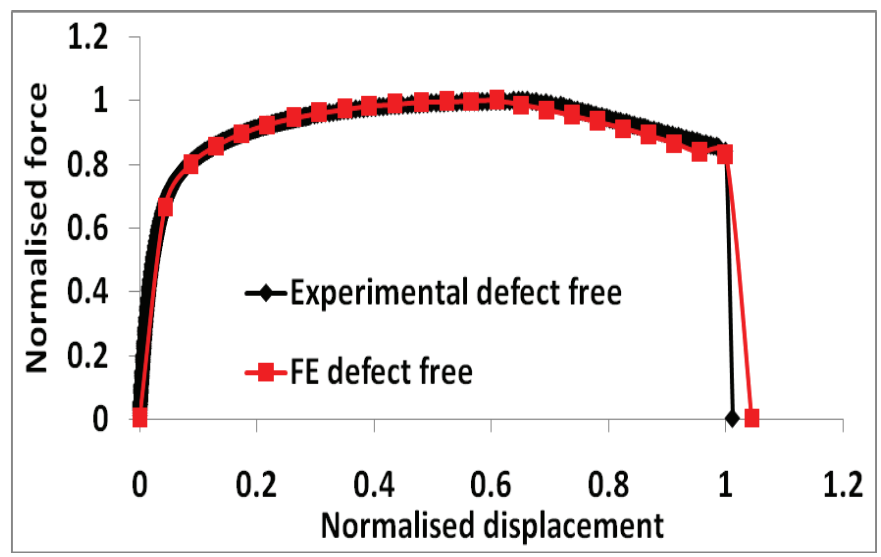

Fig. 3: Force-displacement curves for lamination-free wire 


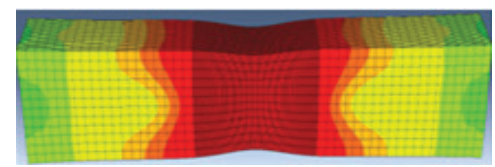

(a) Lamination-free wire specimen

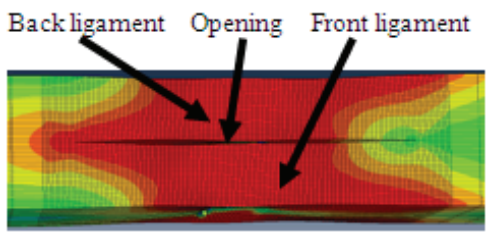

(b) Wire with mid-thickness across-the-width lamination

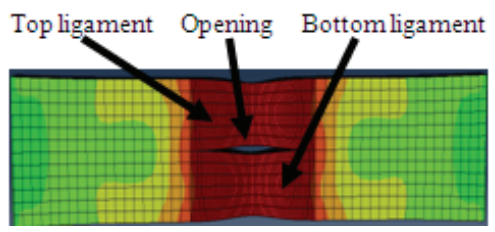

(c) Wire with mid-width across-the-thickness lamination

Fig. 4. FE predicted necked wire models

\section{Discussion}

As shown in Figure 3 and Figures 5(d) and (e), there is a good agreement between the experimental and FE predicted force-displacement curves, and the experimental and FE predicted cup and cone fracture shapes exhibited by the lamination-free wire. This demonstrates the accuracy of the simulation technique employed in this work for the prediction of the tensile and fracture behaviour of lamination-free wire. While the experimental verification of the accuracy of the FE results for the wire with laminations could not be conducted, the good agreement between the FE predicted V-shaped fracture shape with an opening at the pointed end of the $\mathrm{V}$ shape at the location of the lamination shown in Figure 6(d) and the fractured Pre-stressed Concrete Pipe pre-stressing wire with pre-service lamination presented by United States Bureau of Reclamation [1] shown in Figure 6(e) demonstrates the accuracy of the FE simulation in predicting the fracture behaviour of wires with laminations.

As shown in Figure 4(a), the $12 \mathrm{~mm} \times 7 \mathrm{~mm}$ lamination-free wire necked as a single unit without any opening while the wire with laminations necked with an opening between the two ligaments as shown in Figs 4(b) and 4(c). As explained in the earlier work of the Author (see Adewole [12]), the wire with the laminations necked with an opening because the presence of the mid-thickness across-the-width lamination divides the wire into $12 \mathrm{~mm} \times 3.5 \mathrm{~mm}$ front and back ligaments. The front and back ligaments necked separately by shrinking in opposite lateral directions, thereby leaving an opening at the location of the mid-thickness across-the width lamination. Similarly, the presence of the mid-width across-the-thickness lamination divides the wire into $7 \mathrm{~mm} \times 6 \mathrm{~mm}$ top and bottom ligaments with the front and back ligaments necking separately by shrinking in 
opposite vertical directions, thereby leaving an opening at the location of the mid-width across-the thickness lamination.

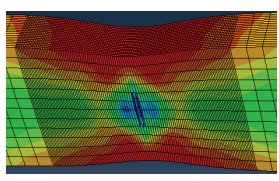

(a) Flat fracture initiation
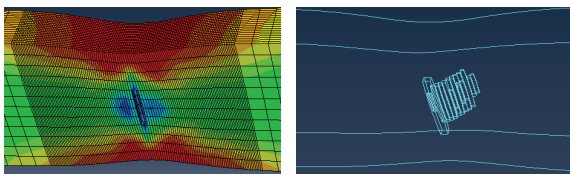

(b) Flat to slant fracture transition

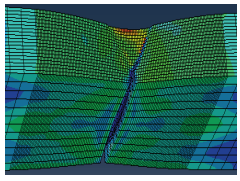

(c) Propagated flat and slant fracture

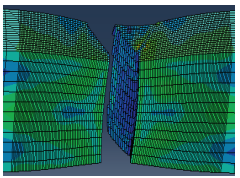

(d) FE fractured wire

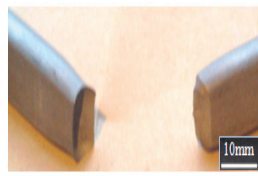

(e) Experimental fractured wire

Fig. 5. Fracture process sequence in lamination-free wire

As shown in Figure 5, the FE predicted cup and cone fracture process for the lamination-free wire follows the well know cup and cone fracture process sequence which begins with a flat fracture initiation at the center of the wire (Figure 5a), followed by flat to slant fracture transition (Figure $5 \mathrm{~b}$ ), and the propagation of the flat and slant fractures (Figure 5c), leading to the cup and cone fracture shown in Figure 5d. Also, as shown in Figs 5(a) to (d), the lamination-free wire fractured as a single unit without any opening and with a fracture initiation occurring at only one location, which is at the center of the entire $12 \mathrm{mmx} 7 \mathrm{~mm}$ single wire unit. As shown in Figure 6(a), the fracture process of the wire with the mid-thickness across-the-width lamination started with two flat fracture initiations simultaneously, one at the center of each of the $12 \mathrm{~mm} \times 3.5 \mathrm{~mm}$ front and back ligaments. The initiated flat fracture propagated vertically along the width of the wire/wire ligaments and transited to slant fractures on both sides of the propagated flat fracture at the outer edges of both the front and back ligaments as shown in Figure 6(b). This was followed by the simultaneous propagation of the flat fracture at the center of the front and back ligaments along the width of the wire/ligaments, and the propagation of the slant fracture across the outer edges of the front and back ligaments along the wire/ligaments' thickness as shown in Figure 6(c), leading to the complete fracture of the wire with a V-shaped fracture surface with opening as shown in Figure 6(d). The completely fractured wire with the mid-thickness across-the-width lamination in Figure 6(d) exhibits a V-shaped fracture shape because the front and back ligaments fractured separately with a negative and a positive slopes respectively. The opening at the pointed end of the V-shaped fracture exhibited by the wire at the location of the mid-thickness across-the width lamination, as earlier stated, resulted from the shrinking of the front and back ligaments in opposite lateral directions due to necking. 


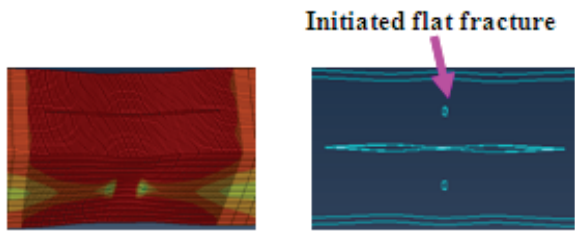

(a) Flat fracture initiation in front and back ligaments

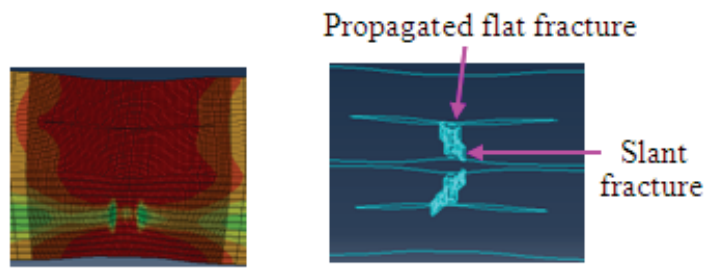

(b) Flat to slant fracture transition in front and back ligaments
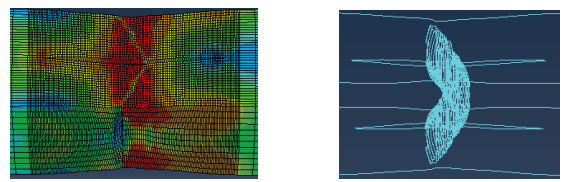

(c) Propagated flat and slant fracture in front and back ligaments

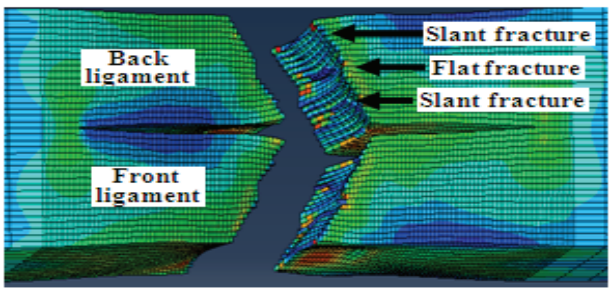

(d) Fractured model of wire with mid-thickness lamination

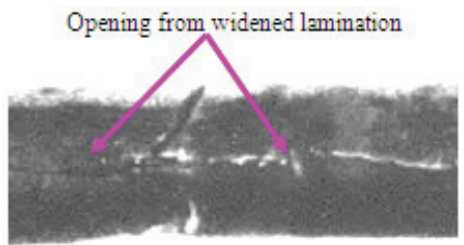

(e) Fractured wire specimen obtained from US Bureau of Reclamation, (1994) [1].

Fig. 6. Fracture process sequence in wire with mid-thickness across-the-width lamination 

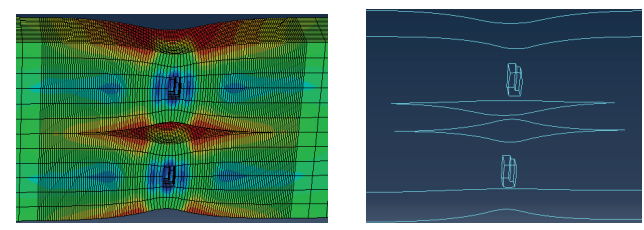

(a) Flat fracture initiation in top and bottom ligament
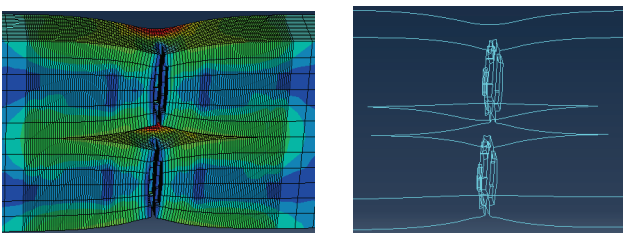

(b) Propagated flat fracture in top and bottom ligament
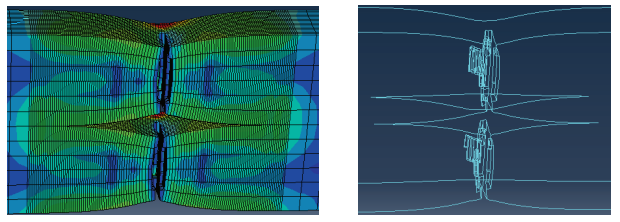

(c) Flat to slant fracture transition in top and bottom ligament
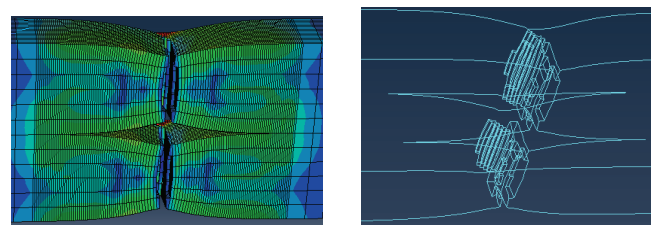

(d) Propagated slant fracture in top and bottom ligament

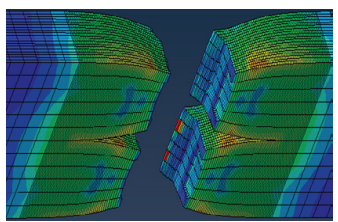

(e) competely fractured wire with mid-width lamination

Fig. 7. Fracture process sequence in wire with mid-width across-the-thickness lamination 
As shown in Figure 7(a), the fracture process of the wire with the mid-width acrossthe-thickness lamination started with two flat fracture initiations simultaneously, one at the center of each of the $7 \mathrm{~mm} \times 6 \mathrm{~mm}$ top and bottom ligaments. The initiated flat fracture propagated vertically across the width of the top and bottom ligaments (width of the wire) simultaneously as shown in Figure 7(b) and transited to slant fractures at the outer edges of both the top and bottom ligaments simultaneously as shown in Figure 7(c). The slant fracture propagated across the outer edges of the top and bottom ligaments simultaneously as shown in Figure 7(d), leading to the complete fracture of the top and bottom ligaments simultaneously as shown in Figure 7(e). The completely fractured wire with the mid-width across-the-thickness lamination in Figure 7(e) exhibits a cup and cone fracture shape with an opening at the middle of the width of the wire where the mid-thickness across-the-width lamination is located. The opening at the mid-width of the cup and cone fracture shape where the mid-width across-the-thickness lamination is located as earlier stated resulted from the shrinking of the top and bottom ligaments in opposite vertical directions. There is more plastic deformation in the centre of the smaller ligaments in this case when compared to the larger ligaments when the lamination is turned through ninety degrees discussed previously. The stress concentration effects of corners have a relatively small effect on the fracture initiation.

\section{Conclusion}

This paper presents the numerical prediction of the effects of lamination orientations on the fracture behaviour of typical rectangular steel wires with laminations used for civil engineering applications using finite element analysis. The investigation covered the effect of the longitudinal mid-thickness across-the-width and mid-width across-thethickness linear crack-like laminations on the fracture behaviour of rectangular wires. FE analysis revealed that the presence of longitudinal mid-thickness across-the-width and mid-width across-the-thickness line-type/crack-like laminations has the same effects on the fracture process sequence and the fracture behaviour of both wire sizes. FE analysis also revealed that the presence of longitudinal mid-thickness across-the-width divides the wire into front and back ligaments while the presence of the mid-width across-the-thickness laminations divides the wire into top and bottom ligaments. FE analysis revealed that the lamination-free wire fractured as a unit while each of the front and back ligaments of the wire with mid-thickness across-the-width lamination and each of the top and bottom ligaments of the wire with the mid-width across-thethickness laminations fractured separately. FE analysis further revealed that the fracture process of the wire with the mid-width across-the-thickness lamination started with two flat fracture initiations simultaneously, one at the center of each of the top and bottom ligaments. Similarly, the fracture process of the wire with the mid-thickness acrossthe-width lamination started with two flat fracture initiations simultaneously, one at the center of each of the front and back ligaments. 
FE analysis also revealed that the presence of the mid-thickness across-the-width lamination changes the cup and cone fracture shape exhibited by the lamination-free wire to a V-shaped fracture shape with an opening at the bottom/pointed end of the $\mathrm{V}$-shape at the location of the mid-thickness across-the-width lamination. The wire with the mid-thickness across-the-width lamination exhibits a V-shaped fracture shape because the front and back ligaments fractured separately with a negative and a positive slopes respectively. The opening at the pointed end of the V-shaped fracture shape exhibited by the wire at the location of the mid-thickness across-the width lamination resulted from the shrinking of the front and back ligaments in opposite lateral directions. FE analysis also revealed that the presence of the mid-width across-the-thickness lamination changes the cup and cone fracture shape of the lamination-free wire to a cup and cone fracture shape with an opening at the location of the mid-width across-the-thickness lamination. The opening at the mid-width of the cup and cone fracture shape where the mid-width across-the-thickness lamination is located resulted from the shrinking of the top and bottom ligaments in opposite vertical directions

The FE fracture behaviour prediction approach adopted in this work provides an understanding of the fracture process, fracture process sequence and the fracture behaviour of wires with longitudinal mid-thickness across-the-width and mid-width across-thethickness laminations. The FE fracture behaviour prediction approach adopted in this work serves as a tool for the investigtion of the effects of longitudinal mid-thickness across-the-width and mid-width across-the-thickness laminations on the fracture behaviour of rectangular wires which cannot be conducted experimentally. This is due to the fact that it is not possible to machine longitudinal mid-thickness across-the-width and mid-width across-the-thickness laminations into the wire specimens.

\section{REFERENCES}

1. United States Bureau of Reclamation. "Pre-stressed Concrete Pipe Failure Jordan Aqueduct, Reach 3" 1994. All U.S. Government Documents (Utah Regional Depository), Paper284. http://digitalcommons. usu.edu/govdocs/284, assessed on 20/04/2014.

2. K. M. Mahmoud, "Fracture strength for a high strength steel bridge cable wire with a surface crack", Theoretical and Applied Fracture Mechanics, vol 48(2), 152-160, 2007.

3. J. Toribio, and F. J. Ayaso, "Anisotropic fracture behaviour of cold drawn steel: a materials science approach", Materials Science and Engineering, vol A343, 265-272.

4. J. Toribio, and F. J. Ayaso, "Fracture Performance of Progressively Drawn Pearlitic Steel under Triaxial Stress States", Materials Science, vol. 37(5), 70-717, 2001.

5. J. Toribio, and A. Valiente, "Failure analysis of cold drawn eutectoid steel wires for pre-stressed concrete", Engineering Failure Analysis, 13 (3), 301-311, 2006.

6. G. F. Vander Voort, "Visual Examination and Light Microscopy", ASM Handbook, vol. 12: Fractography, 91-165, 1987, ASM web. www.asminternational.org, Assessed $15^{\text {th }}$ June, 2014.

7. S. Peet, and A. Wilde, "Laminations-origin, detection and assessment", Congreso Internacional de Ductos, 14-16 November, 2001, Merida Yucatan, Mexico. 
8. K. K. Adewole, and S. J. Bull, "Effectiveness of the reverse bending and straightening tests in detecting laminations in wires for civil engineering applications", Archives of Civil Engineering, 59(4), 423-439, 2013.

9. K. K. Adewole, "Identification of Appropriate Micromechanical Fracture Model for Predicting Fracture Performance of Steel Wires for Civil Engineering Applications", Global Journal of Researches in Engineering: Civil And Structural Engineering, 13(3), 24-33, 2013.

10. K. K. Adewole. Numerical prediction of the effects of longitudinal crack-like laminations on the tensile properties of wires, Journal of Civil Engineering and Management, 2014, in press.

11. K. K. Adewole. Appropriate Mesh Design for Predicting Complete Fracture Behavior of Wires for Civil Engineering Applications", American Society of Civil Engineers Journal of Materials in Civil Engineering, 2014, DOI: 10.1061/(ASCE)MT.1943-5533.0001064, in press.

12. K. K. Adewole. Numerical prediction of the effects of longitudinal crack-like laminations on the tensile properties of wires, Journal of civil engineering and management, doi:10.3846/13923730.2014.91409, in press. 